\title{
Alguns adjuntos temporais com especial relevância para a tradução inglês-português: o caso de since-desde e expressões afins ${ }^{1}$
}

\author{
Telmo Móia \\ Universidade de Lisboa \\ Centro de Linguística da Universidade de Lisboa
}

\begin{abstract}
This paper addresses the semantic analysis of polyvalent temporal adjuncts headed by (mainly) English since and Portuguese desde, and problems in translation from English to Portuguese. Four semantic values of the single operator since are considered (the second and fourth of which are not normally considered autonomously in the English literature) - durative location, derived durative location (in association with adjunct-triggered Aktionsart shift), simple inclusive location and temporal circumscription of quantification. Furthermore, the typically monovalent phrase ever since and the bivalent phrase long since are also taken into account. The fact the Portuguese desde - contrary to English since - is not normally associated with simple inclusive locations is the source of many translation problems. Other interesting grammatical issues, involving long since, are also addressed. The translation data is obtained from the website linguee.com (where six different types of problems are found), and the semantic analysis is made with the logic of the Discourse Representation Theory, elaborating on my previous work, Móia (2000).
\end{abstract}

Keywords: temporal adjuncts, since, translation, semantic interpretation, grammatical anomaly

Palavras-chave: adjuntos temporais, desde, tradução, interpretação semântica, anomalia gramatical

\section{Introdução}

Este trabalho visa explorar - numa perspetiva de gramática contrastiva e com foco em questões de tradução - a expressão de valores temporais através (essencialmente) de adjuntos temporais com o conector inglês since e a sua contrapartida prototípica em português, desde. Como veremos, estes adjuntos formam um grupo que surge com alguma frequência associado a problemas de tradução (a par de pelo menos dois outros grupos interessantes, cuja análise não é possível incluir aqui, nomeadamente: (i) adjuntos com até e until, na correspondência típica, mas com casos, potencialmente indutores de confusão na tradução, de not until a equivaler a só depois ou não antes e de até a equivaler a by, por exemplo; (ii) adjuntos com háa e agolbefore, de um lado, $h a_{2}$ e for, de outro lado, nas correspondências típicas, com possíveis confusões entre si em traduções $^{2}$ ). As possíveis dificuldades da tradução resultam de não haver uma correspondência biunívoca perfeita entre operadores das duas línguas, observando-se, por exemplo, situações em que since corresponde a entretanto ou depois, ou em que desde corresponde a for.

Será feita uma análise de base semântica dos diferentes valores que os operadores relevantes podem assumir - com destaque para quatro deles -, usando a linguagem da Teoria da Representação do Discurso (cf. Kamp \& Reyle, 1993; Móia, 2000). Acredito que esta análise gramatical poderá auxiliar potenciais

\footnotetext{
${ }^{1}$ Este trabalho foi financiado com verbas do projeto estratégico do Centro de Linguística da Universidade de Lisboa UIDB/00214/2020.

${ }^{2}$ Importa distinguir dois conectores equivalentes formados com o verbo haver (tipicamente gramaticalizado na forma há, ainda que possa surgir também na forma havia - cf. e.g. Móia, 2011a): há 1 é um conector que introduz expressões denotadoras de intervalos estruturalmente complexas (possivelmente coincidentes superficialmente com adjuntos de localização temporal), como há dois anos (三 two years ago), em o problema data de há dois anos (atrás) ou o Paulo casou há dois anos (atrás); há 2 é um conector que introduz expressões de duração ancorada, como há dois anos (三for two years), em o Paulo mora em Roma há dois anos.
} 
tradutores a discriminar subtilezas de interpretação e contribuir por essa via para aumentar a qualidade da tradução. Será feita uma ilustração - comentada e relativamente extensa - dos principais problemas identificados no corpus da página linguee.com. Observo, desde já, que esta página contém exemplos tanto de português europeu como de português brasileiro, mas que esse facto não tem importância, já que - tanto quanto consigo apreciar - não há diferenças entre as duas variedades no que respeita às questões específicas de uso do desde que serão apreciadas.

\section{Desde vs. since - correspondências e disparidades}

As expressões temporais com desde são tipicamente equivalentes a expressões inglesas com since:

(1) O rio está congelado desde novembro.

The river has been frozen since November.

(2) Demitiram-se três funcionários de topo desde novembro. Three top officials have resigned since November.

Porém: (i) em certos casos, since não admite a tradução com desde, correspondendo antes ao advérbio entretanto (ou a expressões com depois e afins) - cf. (3); (ii) noutros casos ainda, desde (e.g. nas combinações desde há) não é naturalmente traduzível por since, sendo mais natural a sua correspondência a for - cf. (4) ${ }^{3}$ :

(3) This initiative was very much identified with a coordinator who has since left the school. A iniciativa estava muito ligada a um coordenador que entretanto deixou a escola.

(4) This rainforest hasn't changed for sixty million years. Esta floresta tropical húmida não sofre alterações desde há sessenta milhões de anos (atrás).

\subsection{Ilustração de problemas de tradução de since temporal}

Algumas diferenças entre since e desde estão associadas a problemas de tradução. A construção com since adverbial aplicado a descrições de eventos pontuais, ilustrada em (3) acima, é particularmente saliente. Vejamse (no quadro abaixo) quatro exemplos desta estrutura em traduções da página linguee.com, três dos quais revelam problemas de tradução, de diferentes tipos:

\begin{tabular}{|c|c|c|c|}
\hline $\begin{array}{l}\text { Serbian ambassadors who had } \\
\text { been withdrawn from EU } \\
\text { Member States have since } \\
\text { returned to their posts. }\end{array}$ & $\begin{array}{l}\text { Os embaixadores sérvios que } \\
\text { tinham abandonado os Estados-- } \\
\text { Membros da UE regressaram } \\
\text { entretanto aos seus postos. }\end{array}$ & since $\Rightarrow$ entretanto & tradução OK \\
\hline $\begin{array}{l}\text { The technically-gifted player } \\
\text { hung up his boots in } 2000 \text { and has } \\
\text { since moved into coaching. }\end{array}$ & $\begin{array}{l}\text { O ex-meio-campista pendurou as } \\
\text { chuteiras no ano } 2000 \text { e, desde } \\
\text { então, trabalha como treinador. }\end{array}$ & $\begin{array}{l}\text { since } \Rightarrow \text { desde } \\
\text { (texto traduzido gramatical, mas } \\
\text { com possível alteração do sentido } \\
\text { do original) }\end{array}$ & \multirow[t]{3}{*}{$\begin{array}{l}\text { tradução } \\
\text { problemática }\end{array}$} \\
\hline $\begin{array}{l}\text { [Tirupur (...) buys water for urban } \\
\text { (...) uses from farmers (...) around } \\
\text { the city; as a result these farmers } \\
\text { have since abandoned farming. }\end{array}$ & $\begin{array}{l}{[(\ldots) ;] \text { como resultado, estes }} \\
\text { agricultores abandonaram desde } \\
\text { então sua atividade na fazenda. }\end{array}$ & $\begin{array}{l}\text { since } \Rightarrow \text { desde } \\
\text { (texto traduzido com problemas } \\
\text { de gramaticalidade) }\end{array}$ & \\
\hline $\begin{array}{l}\text { Zambia, like a number of other } \\
\text { countries, has since moved away } \\
\text { from a traditional PRSP }(. . .)\end{array}$ & $\begin{array}{l}\text { A Zâmbia, como vários outros } \\
\text { países, afastou-se de um modelo } \\
\text { tradicional de (...) }\end{array}$ & $\begin{array}{l}\text { since } \Rightarrow \varnothing \\
\text { (informação temporal de since } \\
\text { ignorada, não traduzida) }\end{array}$ & \\
\hline
\end{tabular}

Quadro 1. Ilustração de problemas de tradução do conector temporal since (com exemplos do site linguee.com)

\footnotetext{
${ }^{3}$ Cf. Móia (2003: 52-55) para uma discussão do uso (possível, mas bastante restringido) das expressões since X-TIME ago em inglês
} 
Alguns adjuntos temporais com especial relevância para a tradução inglês-português: o caso de since-desde e expressões afins

Plataformas de tradução automática (como systran.net ou translate.google.com) também documentam traduções problemáticas, em estruturas idênticas - cf. (5) - ou comparáveis - cf. (6):

(5) She has since left school.

$\Rightarrow$ *Ela deixou a escola desde então. (systran.net)

$\Rightarrow$ *Desde então, ela deixou a escola . \| \#Ela já deixou a escola. (translate.google.com)

[cf. ${ }^{\text {OK}}$ Ela deixou a escola entretanto.]

(6) Since then, the industry has collapsed.

$\Rightarrow$ *Desde então, a indústria entrou em colapso. (systran.net = translate.google.com)

[cf. ${ }^{\mathrm{OK}}\{\underline{\text { Depois disso } / \text { Entretanto }\}}$, a indústria entrou em colapso.]

\section{2. (Quatro) Valores de desde e since temporais}

Considerarei, seguindo trabalhos meus anteriores, que as expressões temporais com desde podem estar associadas a três valores semânticos distintos - durativo, durativo derivado e delimitativo da quantificação -, todos eles também permitidos pelas expressões inglesas com since 4 (embora os dois últimos - apresentados e discutidos pela primeira vez em Móia (2000) - não sejam destacados como valores autónomos na literatura sobre o inglês, tanto quanto sei). Tipicamente, as expressões portuguesas não são compatíveis com um quarto valor - inclusivo (simples) -, que é associável às expressões com since (e constitui uma fonte potencial de problemas de tradução, como já vimos).

Farei seguidamente uma brevíssima descrição técnica e ilustração destes quatro valores semânticos, em associação a adjuntos com desde ou since temporais. Posteriormente, os três primeiros serão analisados com mais pormenor, em secções independentes.

A. Localização durativa de situações atélicas (estados ou processos, básicos ou não)

A situação (atélica) descrita na estrutura matriz - ev - cobre todo o intervalo relevante, identificado pelo adjunto com desde - t. Condição formal: $[\mathrm{t} \subseteq \mathrm{ev}]$. Trata-se de uma construção extremamente frequente, quer em português quer em inglês.

(7) O rio está congelado desde novembro.

The river has been frozen since November.

(8) Eles não se tinham encontrado desde que se tinham licenciado ${ }^{5}$.

They had not met since they graduated.

B. Localização durativa derivada de situações atélicas (estados [resultantes])

Trata-se de um valor semelhante ao anterior, mas envolvendo um mecanismo de Aktionsart shift, desencadeado pelo próprio adjunto temporal. Mais especificamente: na frase matriz, descreve-se um evento

\footnotetext{
${ }^{4}$ Refiram-se, como nota prévia, duas particularidades gramaticais das expressões temporais com since que não são partilhadas pelas expressões com desde (mas que não são especialmente relevantes para as questões em apreço neste trabalho): (i) as expressões com since coocorrem normalmente com perfect tenses e não com formas verbais simples (e.g. simple present ou simple past) - three top officials $\left\{{ }^{\mathrm{OK}}\right.$ have resigned / *resigned $\}$ since November vs. três funcionários de topo demitiram-se desde novembro; segundo Huddleston \& Pullum (2002: 697), "since, irrespective of the type of complement, is largely restricted in BrE to occurrence with the perfect" (embora os autores deem um exemplo de uma construção que admite simple tense - it's now nearly a year since he died - e considerem que "AmE allows preterites [i.e. tempos simples do passado] rather more widely”); (ii) since pode ocorrer sozinho, como advérbio (designação tradicional), ou - numa análise alternativa (que prefiro e é perfilhada por e.g. Huddleston e Pullum, 2002: 600-601) - como preposição com um complemento nulo anafórico; diferentemente, desde ocorre sempre com complemento realizado (pronominal/anafórico, nominal ou frásico, três possibilidades também admitidas por since)

${ }^{5}$ Situação atélica na matriz, dada a presença da negação.
} 
pontual (ev), mas o que se localiza temporalmente é o seu estado resultante (s), que se tem de prolongar até ao momento especificado por desde X. Condições formais: $[\mathrm{ev} \supset \subset \mathrm{s}] \wedge\left[\right.$ beg $(\mathrm{t})<_{\text {close }}$ beg $\left.(\mathrm{s})\right] \wedge[$ end $(\mathrm{t}) \mathrm{O} \mathrm{s}]$. Este valor foi identificado em Móia (2000: 283 ss.) e também está disponível para as expressões inglesas com since, como veremos melhor adiante, embora não tenha sido (tanto quanto sei) destacado na literatura sobre o inglês. Não é especialmente frequente, mas ocorre com descrições de certos eventos pontuais (sendo de salientar, entre outros, os identificados por verbos auxiliares aspetuais, como começar a, deixar de, etc.)

(9) Desde que iniciou a terapia com hormonas humanas de crescimento, ele começou a crescer a um ritmo normal.

Since he began therapy with human growth hormone,

he has started to grow at a normal pace. [frase baseada num exemplo de corpus, cf. (26) adiante]

- Evento referido na frase matriz (pontual): $x$ começar a crescer a um ritmo normal.

- Estado resultante localizado (ao longo do intervalo): x estar a crescer a um ritmo normal.

C. Localização inclusiva (simples) de situações (processos culminados e eventos pontuais, tipicamente cf. (10)-(11) -, mas também situações atélicas - cf. (12)).

A situação descrita na estrutura matriz - ev - ocorre algures dentro do intervalo relevante (identificado pelo adjunto com desde) - $\mathbf{t}-$, não importa onde. Condição formal: $[\mathrm{ev} \subseteq \mathrm{t}$ ]. A construção é bastante frequente com since, principalmente com determinadas formas (como veremos adiante), mas não é normalmente possível desde (e a sua tradução é fonte de problemas, como já foi dito).

(10) Messiaen has died since the beginning of this month. (Kamp \& Reyle, 1993: 630)

*Messiaen morreu desde o início do mês.

(11) Since last summer Mary has moved to Paris (ibid.)

* Desde o mês passado, a Mary mudou-se para Paris

(12) Sam has been in Boston since 7.00. (Heny, 1982: 147, Mittwoch, 1988: 206)

${ }^{*} \mathrm{O}$ Sam esteve em Boston desde as 7 horas. [AGRAMATICAL NA LEITURA RELEVANTE, INCLUSIVA] ${ }^{6}$

\section{Delimitação temporal da quantificação}

Nesta construção, a estrutura matriz refere o somatório (total) de situações $-\mathbf{E V}$ - do tipo descrito (ev) que estão incluídas no intervalo relevante (identificado pelo adjunto com desde) - t. Há sempre quantificação sobre situações (direta ou indireta), na frase matriz (cf. expressões em itálico nos exemplos abaixo: seis vezes, três guerras). Condição formal: $\left[\mathrm{EV}=\sum \mathrm{ev}:[\ldots[\mathrm{ev} \subseteq \mathrm{t}] . .].\right]$. É um valor identificado em Móia (2000: $295 \mathrm{ss}$.) e também disponível para as expressões com since, embora não tenha sido destacado como autónomo na literatura sobre o inglês (mas cf. e.g. observações em Quirk et al., 1985:1018, sobre construções semelhantes). A construção é extremamente frequente em ambas as línguas.

(13) Os militares tentaram tomar o poder seis vezes desde que ela se tornou presidente. Military officers have tried to seize power six times since she became president.

(14) As duas nações travaram três guerras desde que se tornaram independentes da Grã-Bretanha. The two nations have fought three wars since they gained independence from Britain.

\footnotetext{
${ }^{6}$ De acordo com as descrições a literatura, a frase (12) é ambígua entre uma leitura durativa e uma leitura inclusiva. Interessa aqui apenas a leitura inclusiva, parafraseável por "o Sam esteve em Boston algures (não importa quando) entre as 7 horas e o momento da enunciação", que corresponderia à tradução portuguesa: o Sam esteve em Boston depois das 7 horas. Huddleston \& Pullum (2002: 709) também mencionam esta ambiguidade, com o exemplo He's been ill again since then.
} 
Alguns adjuntos temporais com especial relevância para a tradução inglês-português: o caso de since-desde e expressões afins

Consideremos agora os três primeiros valores acima referidos separadamente em mais pormenor (nas secções 2,3 , e 4, respetivamente), já que eles são os mais relevantes para as questões de tradução em análise (não tendo sido detetados, nos corpora analisados, problemas relacionados com o quarto valor).

\section{Desde e since como conectores durativos}

Como vimos, estes operadores indicam que uma dada situação (atélica) se prolonga ao longo de todo o intervalo identificado pelo adjunto que eles encabeçam, no que podemos apelidar de localização temporal durativa. Em inglês, este valor pode ser sinalizado de três formas ligeiramente distintas: com o conector simples since e ainda com os conectores complexos ever since e long since. Considerarei seguidamente as duas primeiras possibilidades e deixarei a análise de long since - que suscita questões muito particulares - para a secção 5 .

\subsection{O conector durativo simples since}

Os exemplos do British National Corpus (BNC, doravante) que se seguem e as respetivas traduções, propostas por mim, mostram que os conectores since e desde (durativos) são contrapartidas um do outro.

(15) «The sale of cigarettes to children has been illegal since 1904.» A venda de cigarros a crianças é proibida desde 1904.

(16) «(...) the ethnic Romanian area of Bessar[a]bia (...) has been a source of tension since its 'misappropriation' by the Soviet Union in May 1940.»

A zona etnicamente romena da Bessarábia tem sido uma fonte de tensão desde que a União Soviética se "apropriou indevidamente" dela, em maio de 1940.

(17) «India's troubled northern state of Kashmir, where militants have been waging an armed struggle for independence since 1990, has a new governor. »

O conturbado estado setentrional indiano do Caxemira, onde rebeldes têm estado a travar uma luta armada pela independência desde 1990, tem um novo governador.

(18) «I'm worried about her, she hasn't shed a tear since she had the news (...)» Estou preocupado com ela: ainda não derramou uma lágrima desde que recebeu a notícia.

A construção com since durativo não parece apresentar especiais problemas de tradução (a julgar pela observação de sites com textos traduzidos), se ignorarmos as questões específicas da tradução dos tempos verbais (perfect) do inglês (cf. e.g. has been correspondente a é, em (15), ou a tem sido, em (16)), que são muito curiosas, mas não serão aqui tratadas.

\subsection{O conector durativo ever since}

O inglês, ao contrário do português, pode sinalizar o valor durativo com um conector específico: ever since (que permite evitar ambiguidades com o valor inclusivo ${ }^{7}$ ). Com efeito, ao contrário de since simples, este conector complexo é monovalentemente durativo (com, possivelmente, uma pequena exceção ${ }^{8}$ ).

\footnotetext{
${ }^{7}$ Huddleston e Pullum (2002) dizem que a frase (19) só tem um "continuative reading" ("the situation lasts from her marriage up to now"), o qual está associado, segundo eles, a "imperfective aspectuality" (e não a "perfective aspectuality"). Esta situação contrasta com a da frase (12), com has lived + since simples, que é descrita na literatura como ambígua entre uma leitura durativa e uma leitura inclusiva.

${ }^{8}$ As definições de ever since em dicionários ingleses - em linha com as descrições em gramáticas de referência (e.g. Quirk et al., 1985: 1085; Huddleston \& Pullum, 2002: 141) - dão conta apenas do valor durativo: por exemplo, o Lexico, feito em colaboração com o Oxford Dictionary (lexico.com) parafraseia ever since como "throughout the period since" e o Dictionary by Merriam-Webster (www.merriamwebster.com) como "continually or often from a past time until now; continually from the time in the past when: since". Creio, porém, que em alguns registos de corpora, de um tipo que não vi destacado em dicionários ou na literatura, a locução ever since não pode ser classificada
} 
(19) She has lived in Berlin ever since she married. (Huddleston \& Pullum, 2002: 141) Ele $\{$ vive / tem vivido sempre $\}$ em Berlim desde que casou.

Note-se, marginalmente, que tal como since simples, ever since pode ocorrer com complemento nulo anafórico (i.e. superficialmente como advérbio) - cf. (20) - ou com complemento realizado (pronominal/anafórico, nominal ou frásico) - cf. (21)-(24). É sempre traduzível por desde - que é a sua contrapartida prototípica em português -, sendo apenas de notar que ever since usado sem complementos (como em (20)) é traduzível por desde essa altura, desde então ou formas afins (i.e. desde + complemento anafórico), já que desde não pode ocorrer sozinho, sem complementos realizados.

(20) «Exchange control was introduced in 1930 and has been in force ever since (...).» O controlo cambial foi introduzido em 1930 e \{está / tem estado $\}$ em vigor desde então.

(21) «[In 1977] I bought a (...) book of his paintings. Ever since then I've been a great admirer of his work.»

Em 1977, comprei um livro (...) sobre os seus quadros. Desde essa altura, $\{$ sou / tenho sido $\}$ um grande admirador da sua obra.

(22) «Hitler's capacity for self-deception had been profound ever since the mid-1920s, if not earlier (...).» A capacidade de Hitler de se enganar a si próprio tinha sido enorme desde meados da década de $\underline{1920}$, se não antes.

(23) «The newspapers had been trying him ever since his arrest.» Os jornais tinham andado a julgá-lo desde a sua detenção.

(24) «She had lived alone (...) ever since her husband died of a heart attack (...).» Ela tinha vivido sozinha desde que o seu marido morrera de ataque de coração.

Tal como como since durativo simples, a locução ever since também não parece suscitar especiais problemas na tradução para português. Problemas esporádicos são observáveis, porém, quando o tradutor não capta o sentido durativo, isto é, não percebe que está em causa (como sempre acontece com ever since) uma situação atélica prolongada no tempo. Segue-se um exemplo de uma "tradução falhada" (encontrada no site linguee.com), em que um valor iterativo (atélico) ${ }^{9}$ não foi apreendido, sendo erradamente assumido um valor episódico (télico):

(25) Ever since the historic gathering of thirteen years ago, the Pontifical Council for Interreligious Dialogue has promoted at Rome and Assisi four days of reflection and prayer for world peace and solidarity among peoples.

Decorridos treze anos do histórico encontro de Assis, o Pontifício Conselho para o Diálogo

Interreligioso promoveu em Roma e em Assis quatro dias de reflexão e oração pela paz no mundo e pela solidariedade entre os povos. [TRADUÇÃO INADEQUADA]

Compare-se esta tradução com a que se segue, em que se usa o conector durativo desde associado ao pretérito perfeito composto do verbo eventivo promover (que sinaliza inequivocamente um valor iterativo, atélico):

como genuinamente durativa (pelo menos não é computável com a condição formal $[\mathrm{t} \subseteq$ ev], referida acima em 1.2.) - cf. e.g. «Ever since the summer of the previous year, many horrifying discoveries had been made in the Thames near London.» (BNC; from Murder in old Berkshire: a collection of sudden deaths in and around the old county). Parece tratar-se de uma construção de delimitação temporal da quantificação - cf. quarto valor identificado na secção 1.2 -, que parece poder surgir como ever since, mas só se forem usados quantificadores vagos, como many. As razões para este comportamento excecional dos quantificadores vagos não são claras.

${ }^{9}$ Cf. e.g. Moens (1987) para a associação entre iteratividade e atelicidade. 
Alguns adjuntos temporais com especial relevância para a tradução inglês-português: o caso de since-desde e expressões afins

(25')Desde o histórico encontro de Assis de há treze anos, o Pontifício Conselho para o Diálogo Inter-religioso tem promovido (anualmente) em Roma e em Assis quatro dias de reflexão e oração pela paz no mundo e pela solidariedade entre os povos.

\section{Desde e since como conectores durativos associados a comutação aspetual}

Tanto quanto sei, este valor de since - que designo como durativo derivado - não é destacado como autónomo na literatura sobre o inglês, mas tem múltiplas ocorrências em corpora como o BNC (cf. (26)-(27)) e tem inclusive abonações em dicionários (nos verbetes dedicados a since), como (28). Note-se que na frase matriz desta abonação ocorre o predicado télico pontual lose (its charm) ${ }^{10}$, mas o que se diz que se prolonga até ao momento da enunciação é o estado resultante dele: "o som das vozes das crianças não ter encanto". Sintomaticamente, esta abonação não é destacada como ilustrativa de um valor especial, mas antes integrada numa mesma aceção junto com exemplos de since inclusivo e de since delimitativo da quantificação:

(26) «But Jonas has high hopes: since he began therapy with human growth hormone three years ago, he has started to grow at a normal pace.

Mas o Jonas tem está muito esperançoso: desde que iniciou a terapia com hormonas humanas de crescimento, começou a crescer a um ritmo normal.

(27) «Les, 61, whose wife Tracy (...) is expecting their first child, has stopped smoking since the health scare that led him to quit a show last year.»

Les, de 61 anos, cuja mulher, Tracy, está grávida do seu primeiro filho, deixou de fumar desde que teve um susto com a saúde que o obrigou a cancelar um espetáculo o ano passado.

(28) Since I have become a mother, the sound of children's voices has lost its charm.

(English Cobuild for Advance Learners, 3. ${ }^{\mathrm{a}}$ ed., 2001: 1452)

Desde que me tornei mãe, o som das vozes das crianças perdeu o seu encanto.

Em relação à tradução inglês-português, importa sublinhar que, em certos casos, com since e predicados télicos, podem surgir dúvidas de interpretação entre se se pretende (i) uma leitura inclusiva, equivalente a "algures depois de X" (não traduzível por desde); ou (ii) uma leitura durativa derivada, grosso modo equivalente a "logo a seguir a X", traduzível por desde, se os predicados télicos forem compatíveis" ${ }^{11}$. Veja-se o seguinte exemplo do BNC:

(29) «Since last playing in the NBA, Hudson has started his own record label (...). »

A leitura inclusiva - que parece ser a pretendida - não é traduzível com desde (podendo usar-se e.g. depois ou mais tarde):

(i) Hudson deixou de jogar para a NBA, tendo \{mais tarde / depois \} criado a sua própria marca.

(i') Hudson criou a sua própria marca depois de ter deixado de jogar para a NBA. /

Depois de ter deixado de jogar para a NBA, Hudson criou a sua própria marca.

A leitura durativa derivada pode usar desde, ainda que, neste caso, os verbos criar e lançar não permitam facilmente a construção - cf. (ii) -, sendo melhor optar por uma frase com leitura durativa comum, traduzindo

\footnotetext{
${ }^{10}$ De acordo com Kamp e Reyle (1993: 631, sublinhado meu), "since $\alpha$ characterizes the location of the eventuality described by the nonperfect VP [neste caso, lose its charm]." Curiosamente, neste caso (ao contrário do que acontece na generalidade dos casos), o estado resultante tem de ser diretamente considerado na interpretação.

${ }^{11}$ A diferença entre as duas leituras pode ser neutralizada se houver um valor de posterioridade (do tipo de (i)) muito próxima, induzido contextualmente (como é possível e.g. na paráfrase de (29)(i') acima).
} 
(livremente) o predicado télico por um predicado atélico - cf. (ii') - ou, alternativamente, por uma frase com o conector assim que - cf. (iii):

(ii) ??Hudson criou/lançou a sua própria marca desde que deixou de jogar para a NBA.

(ii') Hudson tem gerido a sua própria marca desde que deixou de jogar para a NBA.

(iii) Hudson criou/lançou a sua própria marca assim que deixou de jogar para a NBA.

Em relação ao uso do conector português desde como durativo derivado, importa fazer duas observações. Em primeiro lugar, com certos predicados pontuais as construções são bem aceites, mas com outros podem surgir dúvidas ou mesmo rejeição (cf. Móia, 2000):

(30) a. O Pedro começou a andar com uma ligadura elástica desde que fez uma tendinite no pé.

b. ??/*O Pedro \{recorreu a / colocou $\}$ uma ligadura elástica desde que fez uma tendinite no pé.

Em segundo lugar, há alguma variabilidade nos juízos de aceitação de construções durativas derivadas com desde (que se afigura como uma possível "área crítica do português"), o que pode naturalmente afetar a tradução. As construções (do CETEMPúblico) a seguir têm, em minha opinião, algum grau (ainda que pequeno) de marginalidade, com os predicados específicos que estão destacados a negrito, admitindo-se que diferentes falantes possam oscilar entre a aceitação ou algum grau de rejeição.

(31) «Desde que foram anunciadas as novas provas que ligam a BSE à doença nos humanos que o Comissário (...) decidiu sublinhar a importância do embargo de sete meses à carne bovina proveniente da Grã- Bretanha.» (ext93745-soc-96b-2)

[vs. ${ }^{\mathrm{OK}}$ Desde que foram anunciadas as novas provas (...) que o comissário tem sublinhado...]

(32) «Desde que se zangou com os filhos, (...) Mrs. B refugiou-se num canto do armazém, onde gere um negócio de carpetes próprio.» (ext53657-eco-95a-2)

[vs. ${ }^{\text {OK }}$ Desde que se zangou com os filhos, Mrs B. está refugiada...]

(33) «Desde que a Arménia se tornou independente (...), a Turquia reiniciou o processo de normalização de relações.» (ext176560-pol-92a-1)

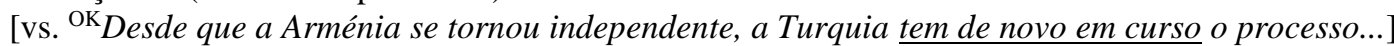

(34) «Desde que foi conhecida a intenção de regular a instalação e funcionamento das «bombas», (...) a Anarec acusou o Governo de ser «mais papista que o papa», ao optar por «rigores excessivos» (...).» (ext180837-eco-95b-2)

[vs. ${ }^{\text {OK }}$ Desde que foi conhecida a intenção..., a Anarec tem andado a acusar o Governo...]

Em contraste, construções com descrições estativas equivalentes (e, portanto, com leitura durativa comum) parecem ser consideradas muito mais naturais - cf. sequências entre parênteses retos a seguir a cada exemplo. Assim, numa tradução, creio que será sempre preferível optar por construções deste tipo, nos casos em que o uso de desde durativo derivado seja de gramaticalidade duvidosa.

Há ainda que considerar - como notei em Móia (2000) - que o valor durativo derivado está, pelo menos em português, intrinsecamente associado à existência de um valor de causalidade (ou afim) entre a situação descrita na matriz e a situação descrita no adjunto (que tem normalmente um complemento frásico). Com efeito, na ausência desse valor, as construções são sentidas como estranhas. Compare-se:

(35) a. O Paulo deixou de fumar desde que o médico lhe disse que corria sérios riscos de vida.

b. ??O Paulo deixou de fumar desde \{o dia 1 de março / quarta-feira\}. [SEM CONTEXTO PRÉVIO]

A questão que discutirei a seguir - porque ela é relevante para questões de tradução discutidas adiante (na secção 5, sobre long since) - é se, contra a generalização acima, são bem aceites pelos falantes construções (de 
Alguns adjuntos temporais com especial relevância para a tradução inglês-português: o caso de since-desde e expressões afins

localização durativa derivada) em que o adjunto tem um valor estritamente temporal, no caso específico de uso da forma (com quantificação vaga) desde há muito (tempo $)^{12}$ - compare-se (35b) com (35c) a seguir:

(35) c. ?O Paulo deixou de fumar desde há muito tempo.

Creio que a gramaticalidade plena deste tipo de frases não é consensual, mas admito que a sua estranheza é menor. Aparentemente, o uso de desde há muito (tempo) em localizações durativas derivadas afigura-se como mais uma "área crítica", havendo variabilidade de juízos dos falantes e um número significativo de registos em corpora de texto jornalístico. O corpus CETEMPúblico tem 1018 registos da sequência desde há muito, associada na maior parte dos casos (como é canónico) a valores durativos comuns ou delimitativos da quantificação. Feita uma análise de parte dos dados (os primeiros 150 registos), a sua associação a valores durativos derivados corresponde a $10 \%$ (15/150 registos), sendo pois a taxa de ocorrência da construção relativamente elevada. Seguem-se quatro exemplos do corpus (onde a construção canónica gramaticalmente inquestionável seria com há muito em vez de desde há muito ou, alternativamente, com uso de uma descrição atélica na frase matriz, de modo a poder manter desde há muito numa leitura durativa comum - cf. sugestões de redação alternativa dadas entre parênteses retos para cada caso):

(36) «É que, como comunista, desde há muito se assumiu como "filho adoptivo do proletariado".» (ext222746-clt-94b-2) [cf. OK há muito se assumiu ou desde há muito se assume]

(37) «O projecto (...) entrou, desde há muito e com carácter irremediável, em processo de desagregação.» (ext153935-clt-95b-1) [cf. ${ }^{\mathrm{OK}}$ entrou há muito ou está desde há muito (em processo de desagregação)]

(38) «(...) [A medida era] previsível, dado o clima de confiança limitada que se instalara desde há muito entre monsenhor Gaillot e os bispos de França (...).» (ext67680-soc-95a-1) [cf. ${ }^{\mathrm{OK}_{\text {se }} \text { instalara }}$ há muito ou estava instalado desde há muito]

(39) «(...) uma comissão de inquérito (...) testemunhou que o genocídio (...) fora planificado desde há muito pelo regime (...).» (ext201399-pol-94b-2) [cf. OK fora planificado há muito ou estava planificado desde há muito]

\section{Desde e since como conectores inclusivos simples}

\subsection{Questões gerais}

Como vimos, o conector since - ao contrário de desde (nas situações mais comuns) - pode indicar que uma dada situação (télica episódica) está incluída no intervalo por ele identificado, no que apelidei de localização inclusiva simples (e que não deve ser confundida com as construções que apelidei de delimitação temporal da quantificação ${ }^{13}$ ).

\footnotetext{
${ }^{12}$ Creio que com quantificação precisa em vez de vaga, a sensação de agramaticalidade é mais forte:

(i) ??O Paulo deixou de fumar desde há dez anos (atrás). nestes contextos, desde é uma tradução perfeitamente natural para since.

A. Presença de verbos de tipo grow / crescer, aumentar e afins (quantificação de graduação)

(i) A empresa cresceu muito desde que mudou de instalações, o ano passado.

(ii) «That was five years ago. Since then Citigate has grown substantially (...). » (BNC)

Isso foi há cinco anos. Desde essa altura, a Citigate cresceu imenso (...)

B. Coordenação copulativa associada a uma implicatura de enumeração exaustiva

(i) Desde que chegaram a Paris, já foram ao Louvre e ao Pompidou e já andaram de barco no Sena.
}

${ }^{13} \mathrm{O}$ conector desde inclusivo simples (que está tipicamente associado a anomalia gramatical) pode ser confundido com o conector desde que encabeça adjuntos de delimitação temporal da quantificação, em algumas estruturas que têm plena aceitação. Destaco 5 contextos (AE abaixo), em que a presença da quantificação relevante não é porventura tão evidente como nos exemplos dados na secção 4 , com quantificadores do tipo $n$ vezes ou $n \mathrm{~N}^{\prime}$ (cf. Móia, (2000), para uma análise formal e uma descrição mais pormenorizada). Crucialmente, 
(40) «(...) they (...) were failed by their original solicitor when they bought their flat, a solicitor who has since died. » (BNC) eles foram enganados pelo seu primeiro advogado quando compraram o apartamento, advogado esse que morreu $\left\{\right.$ * desde então $/{ }^{O K}$ entretanto $\}$

- situação localizada (ev): a morte do advogado

- intervalo de localização $(\mathbf{t})$ : intervalo entre a compra da casa referida e o momento de enunciação da frase (since dêitico)

- relação de localização: $[\mathbf{e v} \subseteq \mathbf{t}]$ (inclusiva)

Vários dicionários de inglês destacam este valor de since, distinguindo-o do valor durativo. Por exemplo, o Collins Cobuild. English Dictionary for Advance Learners (3. ${ }^{a}$ ed., 2001), apresenta o valor durativo como aceção 1, no verbete de since, e distribui o valor inclusivo - que associa aos valores de after e later in time pelas aceções 2 , como preposição ou conjunção (i.e. com complementos realizados), e 3, como advérbio (não o distinguindo também - como se percebe pelas abonações - do valor delimitativo da quantificação e do valor durativo derivado, que aqui considero separáveis).

A observação de corpora (nomeadamente o BNC) mostra que o valor inclusivo simples está frequentemente associado ao uso do since sem complementos realizados, geralmente colocado entre o auxiliar have e o particípio passado (sequência [have since $\mathrm{PP}])^{14}$ :

(41) «She last spoke to the priest five months before the birth and has since lost her teaching job. » A última vez que ela falou com o padre foi cinco meses antes do parto e perdeu o emprego $\{$ *desde essa altura / OKentretanto $\}$

(42) «Tessa (...) was a little nervous as she had just moved from the South (...) but she found the warmest welcome. She has since discovered that (...) Pauline and her colleague Sue are both very knowledgeable and extremely helpful (...).»

[...] Ela descobriu $\left\{{ }^{*}\right.$ desde então $/{ }^{O K}$ entretanto $\}$ que Pauline e a sua colega Sue são ambas pessoas muito informadas e extremamente prestáveis.

(43) «In the 1850s a wing was added to the house (which has since been neatly demolished) (...). » $\mathrm{Na}$ década de 1850 , foi adicionada uma ala à casa (que foi $\left\{*\right.$ desde essa altura $/{ }^{\mathrm{OK}_{\text {entretanto }}}$ cuidadosamente desmantelada) [...]

(ii) «[Allison will be (...) returning to the (...) ground where he began his managerial career 29 years ago with Bath City.] Since then he has been in charge at Plymouth, Crystal Palace, Manchester City and Middlesbrough as well as managing abroad.» (BNC) [...] Desde essa altura, foi treinador do Plymouth, do Crystal Palace, do Manchester City e do Middlesbrough, além de treinador de equipas estrangeiras.

C. Presença de um operador de exclusão (only / só)

(i) Desde 2010, só morreu este elefante no Jardim Zoológico.

(ii) «Cliff's tally of 20 League goals (...) has only been exceeded since 1961 by Mark Bright in 1988 (...). » (BNC) Desde 1961, o recorde de 20 golos na Liga de Cliff só foi ultrapassado, por Mark Bright, em 1988.

D. Dentro de SNs com superlativos ou ordinais

(i) Este é [o livro mais vendido desde 2010] | [Um livro editado pela terceira vez desde 2010] recebeu o prémio.

(ii) «Christian Democrats appear to have suffered their most severe setback in any general election since the Second World War» (BNC) Os democratas-cristãos parecem ter sofrido o seu revés mais severo em eleições gerais desde a II Guerra Mundial.

E. Dentro de N's plurais envolvendo referência à totalidade de objetos com a descrição dada na estrutura nominal relevante

(i) Tudo [o que aconteceu desde essa altura] tem de ser explicado. | A cláusula aplica-se a todos os [contratos (assinados) desde o dia 1].

(ii) «Additionally the new bill applied to all confiscations since May 1939 (...).» (BNC) Além disso, a nova lei aplicava-se a todos os bens confiscados desde maio de 1939.

${ }^{14}$ Ocasionalmente, observa-se ainda a ordem [have PP since] - cf. e.g. «Niki starts with one advantage. A second place will give him the championship. Prost has to finish four points ahead of Lauda to be champion (...). Niki also has, he has written since, 'an indescribable feeling of power. I have never felt as strong as this in my whole life.' (...)» (from The champions of Formula 1 from Fangio to Piquet. Botsford, Keith. London: Stanley Paul, 1988) 
Alguns adjuntos temporais com especial relevância para a tradução inglês-português: o caso de since-desde e expressões afins

Porém, usos de since inclusivo com complementos realizados são referidos na literatura (cf. (10)-(12) acima e (44) abaixo), têm abonações em dicionários (cf. (45)) e têm alguns registos em corpora (cf. (46)-(47)):

(44) I've moved house since you left. (Huddleston \& Pullum, 2002: 709) ??Mudei de casa desde que tu te foste embora.

(45) ... a slight accent she had acquired since I last saw her.

(English Cobuild for Advance Learners, 3. ${ }^{\mathrm{a}}$ ed., 2001: 1452)

??... um ligeiro sotaque que ela tinha adquirido desde que eu a vi pela última vez.

(46) «Here are two more facts to think about, discovered since I wrote the article.» (BNC) *(...) descobertos desde que eu escrevi o artigo

$(47) \ll(\ldots)$ the magazine (...) condemned his Around the World in Eighty Days as an exercise in 'male back-slapping imperialism'. One thing he has discovered since his global circumnavigation, is that it's a big conversation killer.» (BNC)

*[...] uma coisa que ele descobriu desde a sua viagem de circum-navegação [...]

A afirmação de que o conector desde não permite expressar um valor inclusivo simples (e por isso não pode ser usado canonicamente para traduzir since inclusivo) tem de ser modalizada. Essa incompatibilidade existe, de facto, genericamente e pode ser ilustrada pela forte estranheza de frases como (48)-(51):

(48) *O Paulo torceu o pé desde o Natal.

(49) *O Paulo casou com a Ana desde 2005.

(50) */??Esta empresa faliu desde o ano passado.

(51) */??Esta empresa faliu desde (a altura em) que eu me mudei para Paris.

Porém, importa notar duas coisas. Em primeiro lugar, certos contextos parecem reduzir a sensação de estranheza (em particular com desde + um complemento frásico que torne evidente a relevância da fronteira inicial do intervalo), mas - discutivelmente - sem se obter gramaticalidade plena ${ }^{15}$ :

(52) ?O Paulo casou desde que o vi pela última vez. (Móia, 2000: 314)

(52') ?Desde que o vi pela última vez, o Paulo casou.

(53) ?O Paulo esteve com a Ana desde que regressou de férias. (ibid.)

Em segundo lugar, encontram-se por vezes em texto jornalístico português (apesar de serem relativamente raros) exemplos de desde associado a uma localização (que parece ser) inclusiva simples, mostrando que se trata porventura de (mais) uma "área crítica do português", com variabilidade de juízos dos falantes:

(54) «Desde então, conseguiu atingir um ponto de equilíbrio entre as cinco e seis horas diárias de treinos (...), ao abrigo do estatuto de atleta de alta competição.» (ext176594-nd-95a-1) [leitura de entretanto, depois disso?]

(55) «Há meses comecei a escrever baixinho [sic]. Desde então, nos encontros com amigos, aprendi a comunicar através da linguagem gestual.» (ext176594-nd-95a-1)

[leitura de entretanto, depois disso?]

\footnotetext{
${ }^{15} \mathrm{O}$ uso de já (com a leitura de já pelo menos uma vez) pode tornar as construções plenamente aceitáveis - principalmente se interrogativas, como (ii) - ou com um grau mínimo de estranheza - cf. (i) -, mas não assumirei aqui uma posição sobre se estas construções expressam pura localização inclusiva simples (ou se aproximam antes - formalmente - das construções de delimitação temporal da quantificação - cf. Móia 2000: 316, n. 281)

(i) (?)O Paulo já esteve com a Ana desde que regressou de férias. Encontraram-se ontem.

(ii) ${ }^{\mathrm{OK}} \mathrm{O}$ Paulo já tomou banho desde que veio da praia?
} 
(56) «Também me agrada que ele aprenda a língua do seu povo, a sua história e a sua cultura. Desde que aqui chegámos, a minha mãe e o meu pai juntaram-se a nós.» (ext218383-nd-91a-2) [leitura de depois de termos chegado aqui (algures mais tarde)?]

As possibilidades de tradução canónica do conector since inclusivo (prototipicamente na construção [have since $\mathrm{PP}]$ ) são relativamente variadas. Com frequência, o advérbio entretanto adequa-se bastante bem ao sentido pretendido (já que expressa igualmente inclusão num intervalo definido por meios anafóricos e/ou dêiticos - cf. Alves 2003) e é a tradução de maior naturalidade - cf. alternativas em (41)-(43) acima. Outras possibilidades também frequentemente adequadas incluem adjuntos de posterioridade ${ }^{16}$, nomeadamente: (i) conectores depois, após, a seguir a + complementos anafóricos (e.g. isso) ou (ii) advérbios (ou formas afins) como posteriormente, seguidamente, depois, mais tarde. Vejam-se traduções possíveis do exemplo (43) acima, aqui repetido;

(57) In the 1850s, a wing was added to the house (which has since been neatly demolished).

Na década de 1850, foi adicionada uma ala à casa,

... que entretanto (já) foi cuidadosamente desmantelada..

... que $\{$ mais tarde / depois (disso) / posteriormente $\}$ foi / veio a ser $\}$ cuidadosamente desmantelada...

\subsection{Traduções - adequadas e problemáticas - do advérbio since inclusivo (em linguee.com)}

$\mathrm{Na}$ página linguee.com há muitos registos de since com valor inclusivo (na construção [have since PP]). Verifica-se que a tradução é desafiante e fonte de vários tipos de problemas. Identifiquei 6 estratégias utilizadas (identificadas com as letras A a F a seguir): 2 adequadas e 4 problemáticas.

A. Tradução adequada de since inclusivo por entretanto (A1) ou adjuntos de posterioridade (A2), que é a tradução com maior proximidade ao original;

B. Tradução adequada de since inclusivo, mas alterando bastante a estrutura sintática do texto original (i.e. recorrendo a uma tradução mais "livre");

C. Tradução problemática de since inclusivo por adjuntos com desde, que - como vimos - não aceitam canonicamente esse valor, gerando anomalia/agramaticalidade;

D. Tradução problemática de since inclusivo por adjuntos com desde, mudando a Aktionsart no texto de chegada para se adequar a desde, mas alterando - de forma imotivada - o sentido do texto original (de leitura inclusiva para leitura durativa);

E. Tradução problemática de since inclusivo por outros adjuntos temporais que não captam adequadamente o seu valor temporal (e.g. agora);

F. Tradução problemática por eliminação da informação temporal veiculada pelo adjunto (since), que simplesmente é ignorado e não traduzido.

\section{A1: OK (com entretanto)}

(1) Annet (...) learnt early of the partial trisomy of her daughter who has since died.

(2) Serbian ambassadors who had been withdrawn from EU Member States have since returned to their posts.

Annet (...) cedo tomou conhecimento da trissomia parcial da sua filha, que entretanto morreu.

Os embaixadores sérvios que tinham abandonado os Estados-Membros da UE regressaram entretanto aos seus postos.

\footnotetext{
${ }^{16}$ Sintomaticamente, em muitos dicionários ingleses, as paráfrases de since (com o valor relevante) usam operadores deste tipo (after, later in time, subsequently, etc.).
} 
Alguns adjuntos temporais com especial relevância para a tradução inglês-português: o caso de since-desde e expressões afins

(3) The neoliberal ideology has since arrived.

Entretanto, a ideologia neoliberal chegou.

\section{A2: OK (com depois e afins)}

(4) In 1997, people who have since been indicted for war crimes controlled (...) the Army of Republika Srpska.

(5) This factory moved to a less expensive area of the EU and has since moved to Asia.

Em 1997, as pessoas que depois foram acusadas de crimes de guerra controlavam (...) o Exército da Republika Srpska.

A fábrica foi deslocada para uma área menos dispendiosa da UE e posteriormente para a Ásia.

\section{B: OK ("tradução livre")}

(6) (...) they had since left this heavily deforested region.

(...) eles não se encontram mais na região que está muito desmatada. ${ }^{17}$

$\mathrm{C}:$ * (desde inclusivo)

(7) Many of the technologies first developed in the military industry have since been adapted to civilian use (...)

(8) (...) following the termination of anti-dumping measures against Norway in May 2003, and prices had since returned to normal.

(9)

[Tirupur (...) buys water for urban and industrial uses from farmers within 20 to 25 miles around the city;] as a result these farmers have since abandoned farming.

Muitas tecnologias desenvolvidas primeiro no setor militar foram desde então adaptadas para uso civil (...)

(...) que se seguiram à revogação das medidas anti-dumping contra a Noruega em Maio de 2003, tendo os preços desde então voltado aos níveis normais.

[...] como resultado, estes agricultores abandonaram desde então sua atividade na fazenda.

D: $\#^{18}$ (desde durativo)

(10) The technically-gifted player hung up his boots in 2000 and has since moved into coaching.

(11) Sarah Polley has since moved on to roles in mainly independent films (...)

O ex-meio-campista pendurou as chuteiras no ano 2000 e, desde então, trabalha como treinador.

Desde então, Sarah Polley tem se dedicado a desempenhar papéis, principalmente em filmes independentes (...)

\section{E: \# (adjunto inadequado, e.g. agora)}

(12) Research colleagues have since found it in the state of Acre (...)

Colegas de pesquisa agora acharam ele no Acre $(\ldots)^{19}$

\footnotetext{
${ }^{17}$ Registo em PB; em PE (adicionando ainda a vírgula em falta) eles já não se encontram na região, que foi muito desflorestada. Com a estratégia A1: Eles abandonaram entretanto a região, que foi muito desflorestada.

${ }^{18}$ A formulação original have since moved (into / on to) X, destes dois exemplos, não implica necessariamente que a nova situação X (o jogador trabalhar como treinador, ou a atriz desempenhar papéis em filmes independentes), se inicie logo a seguir ao ponto temporal relevante, ao contrário do que é sugerido na tradução (com leitura durativa).

${ }^{19}$ Registo em PB; em PE encontraram-no (em vez de encontraram ele). A formulação original have since found X não implica necessariamente que o objeto $\mathrm{X}$ em causa tenha sido encontrado pouco antes do momento da enunciação, ao contrário do que é sugerido na tradução com agora.
} 


\section{F: \# (adjunto não traduzido)}

\begin{tabular}{l|l|l|}
\cline { 2 - 3 } (13) & $\begin{array}{l}\text { She was taken into care at the kennels in } \\
\text { Marinha Grande and has since found a home. }\end{array}$ & $\begin{array}{l}\text { Foi recolhida no canil da Marinha Grande e } \\
\text { encontrou um lar. }\end{array}$ \\
\hline (14) & $\begin{array}{l}\text { The world has since moved on into what will } \\
\text { likely be a long period of uncertainty. }\end{array}$ & $\begin{array}{l}\text { O nosso mundo entrou, certamente, numa longa } \\
\text { era de incertezas. }\end{array}$ \\
\hdashline $\begin{array}{l}\text { Zambia, like a number of other countries, has } \\
\text { since moved away from a traditional PRSP }\end{array}$ & $\begin{array}{l}\text { A Zâmbia, como vários outros países, afastou-se } \\
\text { de um modelo tradicional de [DERP] (...) }\end{array}$ \\
\hline (...)
\end{tabular}

\section{A locução adverbial (não durativa e durativa) long since}

\subsection{Questões gerais}

A locução adverbial long since (que, como ever since - cf. secção 2.2 -, inclui a forma since na sua composição, ainda que possivelmente ela não seja computada autonomamente) tem especial interesse para a tradução inglês-português. Registos lexicográficos de long since dão conta de dois valores, um não durativo (ou inclusivo) e um durativo (nos termos que aqui estou a utilizar). ${ }^{20}$

(73) long since $_{1}$ : It has long since vanished. | Desapareceu há muito. ${ }^{21}$

(74) long since $e_{2}$ They have long since been friends. | São amigos (desde) há muito.

Note-se, marginalmente, que esta locução adverbial pode ter um valor dêitico (de anterioridade ao momento da enunciação) - como em (73)-(74) - ou um valor anafórico (de anterioridade de um ponto de perspetiva temporal [PPT] discursivamente determinado) - como em e.g. it had long been vanished| desaparecera há muito. Vejamos com mais pormenor cada um dos dois sentidos da expressão.

\section{A. long since não durativo - long since 1}

Nos exemplos mais comuns, long since combina-se com descrições de eventos pontuais, portanto télicos (expressando uma mera ocorrência retrospetiva no tempo, a grande distância do ponto de perspetiva - uma "localização remota", por assim dizer). Equivale à expressão inglesa long time ago. Nesta aceção, não tem qualquer valor de tipo durativo (ou aproximável dos valores de desde) e corresponde tipicamente ao português há/havia muito ((tempo) atrás), entre outros - cf. Quadro 2 adiante:

(16) «The stones of the church are dark with age and the roof has long since gone. » As pedras da igreja estão escurecidas com a idade e o telhado \{há muito que desapareceu / desapareceu há muito (tempo atrás) \}.

\footnotetext{
${ }^{20}$ O Dictionary by Merriam-Webster (www.merriam-webster.com), por exemplo, indica as duas aceções da locução: 1 . 'long ago'; 2 . 'for a long time' (e.g. has long since been a devoted friend). Outros dicionários (e.g. o Collins Cobuild - English Dictionary for Advanced Learners ou o Longman Dictionary of Contemporary English Online [www.ldoceonline.com]) apenas consideram a primeira aceção, que é aparentemente muito mais frequente. O dicionário Longman, curiosamente, apesar de só considerar a primeira aceção, inclui 2 exemplos da segunda nas 8 abonações que apresenta, sem os distinguir (e.g. the Democratic Party has long since taken black voters for granted; (...) he had long since trained himself to tune out all awareness of boys (...)). A maior frequência da aceção 1 é facilmente percetível pela observação dos 521 registos da sequência long since no British National Corpus (e está em linha com as observações sobre registos em dicionários ingleses acima).

${ }^{21}$ A expressão tem uma versão negativa, not long since, traduzível por e.g. não há muito; dois exemplos do BNC: «A very striking example was told me not long since by someone who attended a dinner party in Moscow soon after Gagarin's space trip (...).»; «The doctor had intended to reciprocate with a copy of the Dictionary (...), but on finding it out of print, sent a copy of the Abridgement 'not long since published' (fifth edition, 1767). »
} 
Alguns adjuntos temporais com especial relevância para a tradução inglês-português: o caso de since-desde e expressões afins

(17) «Any normal person would long since have collapsed.» Qualquer pessoa normal ter-se-ia ido abaixo há (já) muito tempo.

(18) «John (...) had long since lost his northern accent and took delight in his appearance as the well-heeled businessman.»

O John \{há/havia\} muito que perdera o seu sotaque do Norte e tinha muito orgulho no seu ar de homem de negócios bem na vida.

\section{B. long since durativo - long since 2}

Noutras estruturas, long since combina-se com descrições de situações atélicas. Neste caso, o valor é de tipo durativo e a tradução pode incluir desde (e.g. na fórmula desde há muito); pode alternativamente usar apenas expressões com há homónimas das que traduzem long since 1 , mas com o valor (distinto) de duração ancorada $^{22}$. Nesta aceção, long since equivale a for a long time (now/then).

(19) «The town outside was long since ruined and empty (...)» Lá fora, a cidade estava (desde) há muito em ruínas e deserta (...)

(20) «Brittany had long since been in some way subject to Normandy.»

Em certo sentido, a Bretanha estava (desde) há muito subjugada à Normandia.

(21) «Nearly forty years ago (...) [E. H. Carr] published a series of broadcast talks called The New Society (long since out of print).» ... ((desde) há muito esgotadas / esgotadas (desde) há muito tempo)

(22) «As a septuagenarian, he made the trip to Ireland (...) to find the Killarney Fern long since believed gone from its single recorded site on Moel Hebog (...).» (...) para encontrar o feto-de-killarney, que (desde) há muito se acreditava ter desaparecido do único lugar em que tinha sido registado, em Moel Hebog.

As possibilidades de tradução canónica do operador long since - no seus dois sentidos (não durativo [long since $_{1}$ ] e durativo [long since 2 ]) - são relativamente variadas. Algumas são comuns aos dois sentidos, outras são exclusivas de um dos sentidos. As principais possibilidades estão resumidas abaixo:

\begin{tabular}{|c|c|c|}
\hline inglês & \multicolumn{2}{|c|}{ português } \\
\hline long since 1 ou $\left[\mathrm{F}_{[+\mathrm{T}} \mathrm{L}\right]$... long since...] & \multirow{2}{*}{$\begin{array}{l}\text { há muito (tempo) } \pm \text { que } \\
\text { havia muito (tempo) } \pm \text { que [SÓ PARAPPT } \\
\text { PASSADO] } \\
\text { também formas com fazer - cf. Móia (2020): } \\
\quad \text { e.g. faz/fazia (já) muito tempo }\end{array}$} & $\begin{array}{l}\text { há muito tempo atrás } \\
\text { muito (tempo) antes [SÓ PARAPPTPASSADO] }\end{array}$ \\
\hline \multirow[t]{2}{*}{$\begin{array}{l}\text { long since }{ }_{2} \text { ou }\left[\mathrm{F}_{[-\mathrm{TÉL}]} \text {... long since...] }\right. \\
\text { [MENOS FREQUENTE] }\end{array}$} & & $\begin{array}{l}\text { desde há muito ((tempo) atrás) } \pm \text { que } \\
\text { [POUCO USADO] desde havia muito (tempo) }\end{array}$ \\
\hline & \multicolumn{2}{|c|}{$\begin{array}{l}\text { Sequências agramaticais ou marginais no PE padrão - cf. Móia (2010, 2011a): } \\
\text { *há muito tempo atrás que, *(desde) havia muito tempo atrás (que) }\end{array}$} \\
\hline
\end{tabular}

Quadro 2. Possibilidades de tradução canónica da locução long since para português

\footnotetext{
${ }^{22}$ Aqui, há é contrapartida de for (duração ancorada) e não de ago/before (identificação de intervalos/localização), como acontece nos casos (16)-(18) (ou seja é $h a_{2}$ e não há , na notação da nota de rodapé 1) - sobre estes dois valores de há em português, cf. e.g. Móia (2006, 2011a,b).
} 


\subsection{Traduções - adequadas e problemáticas - da locução long since (em linguee.com)}

Na página linguee.com há 16 registos da locução long since (todas, sintomaticamente, de long since l). $_{1}$. Mesmo num corpus tão pequeno, verifica-se que a tradução desta expressão pode ser fonte de anomalias, tendo sido identificadas 4 estratégias: 2 adequadas, 1 discutível e 1 problemática.

A. Tradução adequada de long since ${ }_{1}$ por há muito (e variantes), opção com maior proximidade ao original;

B. Tradução adequada de long since ${ }_{1}$ por desde há muito (e variantes), correspondentes a long since ${ }_{2}$; é uma opção viável, desde que se mude a telicidade do predicado relevante (sem se alterar significativamente o sentido da mensagem), numa tradução com menor proximidade ao original;

C. Tradução (de aceitação) discutível de long since ${ }_{1}$ por desde há muito e variantes (correspondentes canonicamente a long since 2 ), com interpretação de tipo durativo derivado (cf. discussão no final da secção 3, acima);

D. Tradução problemática por eliminação da informação temporal veiculada pelo adjunto (long since $_{1}$ ), que simplesmente é ignorado e não traduzido, perdendo-se neste caso a informação de longinquidade.

(23) The roof of the church has long since 1 gone.

O telhado da igreja desapareceu há muito. (tradução tipo A)

A igreja está sem telhado desde há muito. (tradução tipo B)

?O telhado da igreja desapareceu desde há muito. (tradução tipo C)

\#O telhado da igreja desapareceu. (tradução tipo D)

(24) John has long since 1 lost his accent.

O John perdeu há muito o seu sotaque. (tradução tipo A)

O John já não tem desde há muito o seu sotaque. (tradução tipo B)

?O John perdeu desde há muito o seu sotaque. (tradução tipo $\mathrm{C}$ )

\#O John (já) perdeu o seu sotaque. (tradução tipo D)

A: OK (com há muito e afins)

\begin{tabular}{|l|l|l|}
\hline$(25)$ & $\begin{array}{l}\text { We think that it has long since passed its sell-by } \\
\text { date. }\end{array}$ & $\begin{array}{l}\text { Pensamos que já ultrapassou há muito o seu } \\
\text { prazo de validade. }\end{array}$ \\
\hline (26) & $\begin{array}{l}\text { (...) physical combat as a technique of adjusting } \\
\text { racial misunderstandings has long since fallen } \\
\text { into disrepute. }\end{array}$ & $\begin{array}{l}\text { (...) o combate físico, como uma técnica de } \\
\text { ajustamento de mal-entendidos raciais, há muito } \\
\text { tempo caiu em descrédito. }\end{array}$ \\
\hline (27) & $\begin{array}{l}\text { To be sure, it had long since been realized that } \\
\text { many of the people who went around pretending } \\
\text { to be philosophers, (...) }\end{array}$ & $\begin{array}{l}\text { Certamente, já há muito tempo que os homens } \\
\text { se tinham apercebido de que boa parte dos que } \\
\text { circulavam como filósofos, (...) }\end{array}$ \\
\hline
\end{tabular}

\section{B: OK (com desde há muito e afins + mudança de valor de telicidade)}

(28) This involves the fight against crime and terrorism, which have long since moved beyond national frontiers.

Isto diz respeito à luta contra o crime e $\mathrm{o}$ terrorismo, que desde há algum tempo não conhecem fronteiras. ${ }^{23}$

\footnotetext{
${ }^{23}$ Esta tradução tem ainda alguns outros problemas (marginais, relativamente ao que está em apreço): (i) é questionável a equivalência entre moved beyond national frontiers e não conhece fronteiras (se bem que é a mudança de telicidade da expressão predicativa do original [télico moved $\rightarrow$ atélico não conhece] que torna possível o uso de desde, um operador que só traduz long since 2 ); uma opção que parece melhor (mantendo a estratégia B) seria: ... que já desde há muito se trava fora das fronteiras nacionais; (ii) é questionável a opção pelo quantificador algum em vez de muito (que traduz melhor a informação veiculada pelo original); (iii) a concordância do verbo deveria ser no plural, como no original (conhecem, a concordar com o crime e o terrorismo). Numa tradução mais próxima do original, com preservação da telicidade (tipo A), poderíamos ter: ... que há muito ultrapassaram as fronteiras nacionais.
} 
Alguns adjuntos temporais com especial relevância para a tradução inglês-português: o caso de since-desde e expressões afins

\section{C: ? (com desde há muito e afins)}

\begin{tabular}{|l|l|l|}
\hline (29) & $\begin{array}{l}\text { Sweden has long since ceased the distributions of } \\
\text { foodstuffs to the most deprived persons which } \\
\text { were carried out formerly. }\end{array}$ & $\begin{array}{l}\text { Na Suécia, renunciámos desde há muito às } \\
\text { distribuições de géneros alimentícios aos mais } \\
\text { desfavorecidos outrora praticadas. }{ }^{24}\end{array}$ \\
\hline
\end{tabular}

\section{D: \# (adjunto não traduzido)}

\begin{tabular}{|l|l|l|}
\hline (30) & $\begin{array}{l}\text { (...) the period within which [the law] (...) must be } \\
\text { promulgated has long since passed and, } \\
\text { consequently, it has not entered into force. }\end{array}$ & $\begin{array}{l}\text { (...) o período durante o qual [a lei] deveria ter } \\
\text { sido promulgada já passou e consequentemente } \\
\text { não entrou em vigor. }\end{array}$ \\
\hline (31) & $\begin{array}{l}\text { She also recounted that many people has long } \\
\text { since left the village, attracted to the city life in } \\
\text { Lisbon. }\end{array}$ & $\begin{array}{l}\text { Ela também nos contou que muita gente deixou a } \\
\text { aldeia para ir à procura de uma vida melhor na } \\
\text { cidade de Lisboa. }\end{array}$ \\
\hline
\end{tabular}

\section{Conclusões}

Em suma, verificámos que os adjuntos temporais do grupo since-desde estão associados a problemas de tradução inglês-português, sendo de destacar, como indicado na Figura 1 abaixo, três famílias de problemas:

(i) casos de tradução com anomalia gramatical, de dois tipos - (a) tradução de since inclusivo por desde e bem assim (b) de long since não durativo por desde há muito;

(ii) casos de má interpretação de since ou ever since, trocando interpretações durativas e não durativas, também com dois tipos identificados;

(iii) casos de simples eliminação da informação temporal, por não se traduzir o operador relevante (o que podemos conjeturar que talvez se deva a dificuldades de interpretação), documentado em dois casos - com since inclusivo e com long since não durativo.

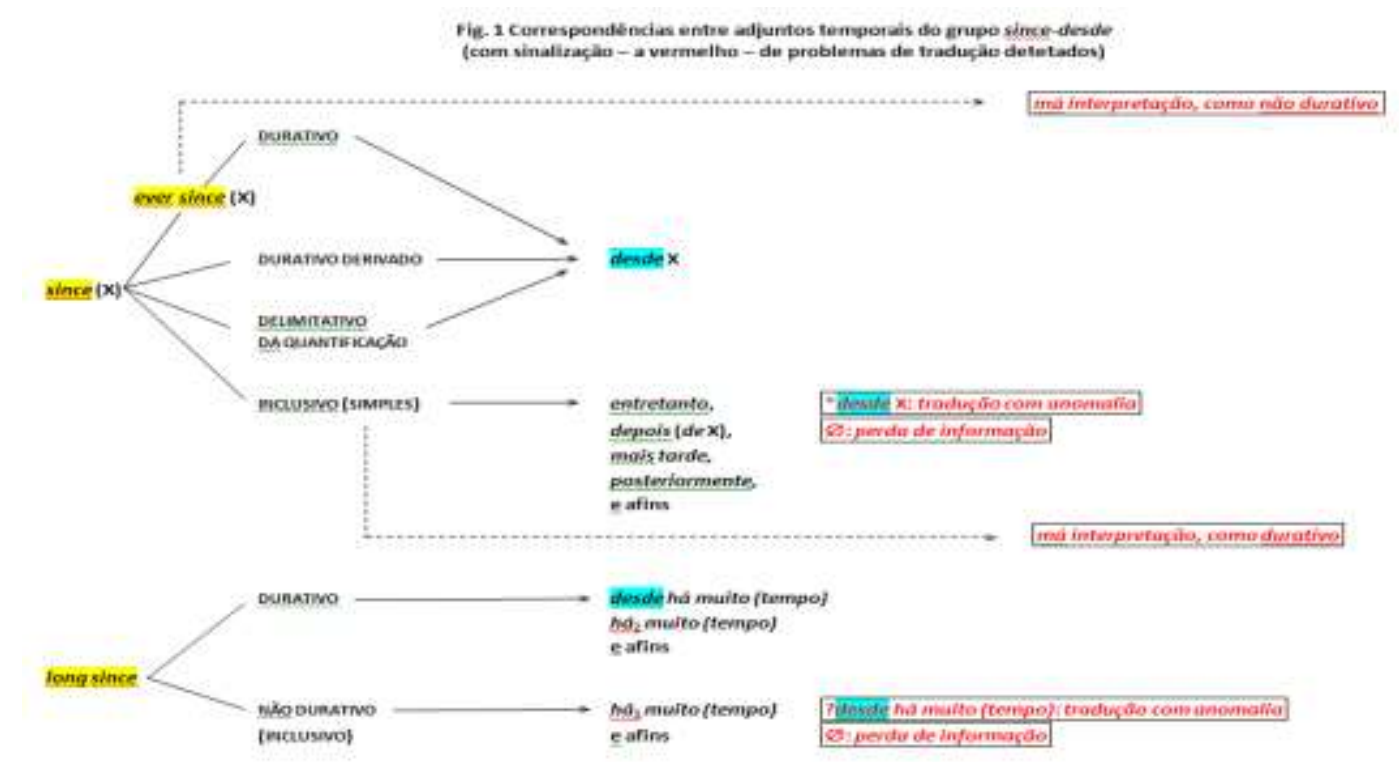

\footnotetext{
${ }^{24}$ Alternativas plenamente canónicas a renunciámos desde há muito às distribuições, seriam renunciámos há muito às distribuições (estratégia A) ou já não fazemos desde há muito as distribuições (estratégia B).
} 


\section{Referências (incluindo dicionários de inglês, corpora e websites de tradução consultados)}

Alves, Ana Teresa (2003) Sobre a Localização Temporal Adverbial Anafórica em Português. Dissertação de doutoramento, Universidade dos Açores, Ponta Delgada.

Heny, Frank (1982) Tense, Aspect, and Time Adverbials. Part II. Linguistics and Philosophy 5, pp. 109-154.

Huddleston, Rodney \& Geoffrey K. Pullum (2002) The Cambridge Grammar of the English Language. Cambridge: Cambridge University Press.

Kamp, Hans \& Uwe Reyle (1993) From Discourse to Logic. Introduction to Modeltheoretic Semantics of Natural Language, Formal Logic and Discourse Representation Theory. Dordrecht: Kluwer.

Mittwoch, Anita (1988) Aspects of English Aspect: on the Interaction of Perfect, Progressive and Durational Phrases. Linguistics and Philosophy 11, pp. 203-254.

Moens, Marc (1987) Tense, Aspect and Temporal Reference. Ph.D. thesis, University of Edinburgh (reproduced by the Centre for Cognitive Science, University of Edinburgh).

Móia, Telmo (2000) Identifying and Computing Temporal Locating Adverbials with a Particular Focus on Portuguese and English Dissertação de doutoramento, Universidade de Lisboa.

Móia, Telmo (2003) On Temporal Constructions Involving Measurement and Counting from Anchor Points Semantic and Pragmatic Issues. In K. M. Jaszczolt \& K. Turner (eds.) Meaning Through Language Contrast, Vol. 1. Amsterdam / Philadelphia: John Benjamins, pp. 45-59.

Móia, Telmo (2006) Portuguese Expressions of Duration and their English Counterparts. Journal Of Portuguese Linguistics 5 (1), pp. 37-73.

Móia, Telmo (2010) Expressões de Medição Temporal: Norma, Variação e Desvio. In. XXV Encontro Nacional da Associação Portuguesa de Linguística. Textos Seleccionados (Lisboa, 22, 23 e 24 de Outubro de 2009). Porto: APL, pp. 623-641

Móia, Telmo (2011a) Expressões Temporais com 'Haver': Gramaticalização e Interpretação Semântica. In. XXVI Encontro Nacional da Associação Portuguesa de Linguística. Textos Seleccionados 2010. Porto 2010. Lisboa: APL, pp. 401-419.

Móia, Telmo (2011b) Sobre a Expressão Lexical da Duração e da Localização Temporal em Português. In. M. Arden, C. Märzhäuser \& B. Meisnitzer (eds.) Linguística do Português. Rumos e Pontes. München: Martin Meidenbauer Verlag,, pp. 251-269.

Móia, Telmo (2020) Predicados Temporais e Gramaticalização em Português. In. B. Meisnitzer \& E. Pustka (orgs.) Zwischen Sprechen und Sprache / Entre Fala e Língua, Berlin: Peter Lang GmbH, pp. 59-81.

Quirk, Randolph et al. (1985) A Comprehensive Grammar of the English Language.London: Longman.

\section{Dicionários de inglês}

Collins Cobuild. English Dictionary for Advance Learners, Glasgow, HarperCollins Publishers 3. a ed., 2001

Dictionary by Merriam-Webster: www.merriam-webster.com

Lexico (feito em colaboração com o Oxford Dictionary): www.lexico.com

Longman Dictionary of Contemporary English Online: www.ldoceonline.com

\section{Corpora}

[BNC] British National Corpus, acessível em https://corpus.byu.edu/

[CETEMPúblico] Corpus cetempúblico 1.7 v. 7.1, acessível em http://www.linguateca.pt/ACDC/

Websites de tradução

www.linguee.com

WwW.systran.net

www.translate.google.com 